\title{
Enhanced antifouling performance of hybrid PVDF ultrafiltration membrane with the dual-mode $\mathrm{SiO}_{2}$-g-PDMS nanoparticles
}

\author{
Haiye Wang, Xinzhen Zhao, Chunju He* \\ State Key Lab for Modification of Chemical Fibers and Polymer Materials, College of \\ Materials Science and Engineering, Donghua University, Shanghai 201620, China \\ *chunjuhe@dhu.edu.cn
}

\begin{abstract}
The polydimethylsiloxane modified silica $\left(\mathrm{SiO}_{2}-\mathrm{g}-\mathrm{PDMS}\right)$ nanoparticles with hydrophilic core and hydrophobic shell are employed for the hybridization modification of PVDF ultrafiltration membrane to improve the separation and antifouling performances. The results suggest the modified $\mathrm{SiO}_{2}$-g-PDMS nanoparticles exhibit improved dispersion and significant amphiphilic characteristics, and the wetting properties of membrane surface and mimetic matrix of hybrid PVDF membranes are distinctly different with the contact angle values at $105^{\circ}$ and $15^{\circ}$, respectively. In addition, the added $\mathrm{SiO}_{2}$-g-PDMS nanoparticles significantly improve the fouling repulsion and fouling release properties of hybrid PVDF membranes, the anti-adsorption ability and water flux recovery ratio (FRR-W) of hybrid PVDF membranes to the two typical pollutants of bovine serum albumin (BSA) and humic acid (HA) have been significantly improved. This article aims to provide a simple way to improve the antifouling performance of PVDF ultrafiltration membrane with dual-mode multi-functional modifier.
\end{abstract}

Keywords: polyvinylidene fluoride, hybrid, dual-model, antifouling 


\section{Introduction}

Membrane technology has been regarded as one of the most economic separation process, and played an increasingly important role in the wastewater treatment and water security. ${ }^{1}$ However, membrane fouling ${ }^{2-4}$ as an inevitable problem in almost all the membrane processes limits the commercial application of membrane technologies. For the ultrafiltration (UF) membrane, the various types of hydrophobic pollutants such as proteins will induce serious irreversible fouling problem to decrease the separation efficiency due to the hydrophobic effect between pollutants and membrane materials such as polyvinylidene fluoride (PVDF), polyether sulfone (PES) and polyvinyl chloride (PVC). Therefore, replacing the fouled membrane module with extra cost is the most commonly used method to solve the membrane fouling problem. As an imperative and urgent scientific issue, the preparation of non-fouling or low-fouling UF membranes has attacked a lot of attention.

The reported literatures suggest that the antifouling UF membranes are required to equip the fouling repulsion and fouling release properties, ${ }^{5,6}$ diminishing the non-specific adsorption between membrane materials and pollutants and high removal probability of pollutants are prerequisite for the UF membrane to exhibit the desired antifouling effect. The fouling repulsion ability is based on the interfacial free energy theory and can be realized by increasing the hydrophilicity of membrane material to inhibit the adsorption of pollutants on membrane surface, ${ }^{7,8}$ and it is generally believed that water molecules can be preferentially adsorbed onto the hydrophilic material surface to form a protective layer to repulse the pollutants molecules in the aqueous separation interface, ${ }^{2,9,10}$ which plays an important role for reducing the adsorption amount of hydrophobic pollutants and the anchoring sites on 
membrane surface. Polyethylene glycol (PEG) based materials, ${ }^{11}$ zwitterion based materials ${ }^{12}$ and hydrophilic nanomaterials ${ }^{13}$ with large specific surface area, high hydrophilicity and vast active groups are the mostly used agents for hydrophilic modification. The fouling release ability ${ }^{14}$ of membrane refers to the scavenging activity to the pollutants adsorbed on the materials interface though proper cleaning method, fluorinated materials and polydimethylsiloxane (PDMS) are considered to be the best antifouling material with release capability due to the ultra-low surface energy. As we all know, PDMS with excellent weather resistance, low surface energy, strong shear resistance, excellent lubricating effect and thermal stability is widely used in antifouling coating. In addition, the great freedom of rotating silicon-oxygen backbone impedes the possibility to release the pollutants with the aid of shear force during cleaning process. Therefore, PDMS can be used as the fouling release material. $^{15}$

In this work, in order to unite the fouling repulsion and fouling release abilities, $\mathrm{SiO}_{2}$-g-PDMS nanoparticles with dual antifouling effect were prepared based on the directional reaction of terminal functional groups, and used to hybrid the PVDF UF membrane. The physical and chemical structure, separation performance and antifouling properties of prepared hybrid UF membranes were all investigated. This article aims to combine the antifouling advantages of hydrophilic nanoparticles and hydrophobic PDMS to provide a novel reference for the hybrid modification.

\section{Experimental}

\subsection{Materials}

Polyvinylidene fluoride (PVDF, MG15) was purchased from Arkema. 
Polydimethylsiloxane with terminal hydroxyl (HO-PDMS-OH, Mw 4000) was purchased from Fangzhou chemical (China). $\mathrm{SiO}_{2}$ nanoparticles $(30 \mathrm{~nm}$ ), trimesoyl chloride (TMC) and humic acid (HA, fulvic acid> 90\%) were purchased from Aladdin chemical Co. Bovine serum albumin(BSA, 67000Da) and polyethylene glycol (PEG, Mw 4000) were purchased from Sinopharm Chemical Reagent Co.(China). Dimethylacetamide (DMAC), n-hexane and toluene were analytical grade. All the reagents were used as received without further purification.

\subsection{Synthesis of $\mathrm{SiO}_{2}$-g-PDMS nanoparticles}

TMC and PDMS were added into $50 \mathrm{ml} \mathrm{n}$-hexane solution with a molar ratio of $1: 2$, and this mixture solution was kept to react for 1 hour under magnetic stirring at room temperature. Then the $\mathrm{SiO}_{2}$ nanoparticles dispersion solution (n-hexane as solvent, ultrasound for 30 minutes) was added to the above solution to react for another $2 \mathrm{~h}$ under stirring. Finally, the nanoparticles were filtered and washed three times using n-hexane, and dried for the further use.

\subsection{Preparation of hybrid PVDF membranes}

All the membranes were prepared using non-solvent induced phase separation (NIPS) method. A certain amount of modified $\mathrm{SiO}_{2}$-g-PDMS nanoparticles were added to the casting solution (PVDF/PEG4000/DMAC $=10 \mathrm{~g} / 4 \mathrm{~g} / 50 \mathrm{~g})$. On the basis of PVDF mass, the added mass ratios of $\mathrm{SiO}_{2}$-g-PDMS nanoparticles were $0 \mathrm{wt} \%, 3 \mathrm{wt} \%, 8 \mathrm{wt} \%$ and $15 \mathrm{wt} \%$, and the prepared membranes were labeled as PVDF, 3\%, $8 \%$ and 15\%, respectively. It should be noted that the nanoparticles and porogen PEG were added together in advance to ultrasound for 30min. In addition, the unmodified $\mathrm{SiO}_{2}$ nanoparticles were used to prepare reference 
sample with added mass ratio of $8 \mathrm{wt} \%$, this sample was named as $\mathrm{R}-8 \%$.

\subsection{Characterization}

The properties of modified $\mathrm{SiO}_{2}$-g-PDMS nanoparticles were characterized with infrared spectroscopy (FTIR, Nicolet 8700, USA), X-ray photoelectron spectroscopy (XPS, Shimadzu AXIS UltraDLD, Japan), thermogravimetric analyzer (TGA, TG 209 F1 Iris, Netzsch) and nanoparticle size and potential analyzer (Nano ZS, Malvern).

The wetting ability of surface and porous matrix of neat and hybrid PVDF membranes were evaluated by water contact angle (CA, OCA40Micro, Germany) at room temperature. The contact angle of the membrane surface can be determined directly, but the contact angle of the porous media cannot be directly tested due to the barrier of skin layer. Therefore, the mimetic porous matrix was prepared by spin coating method and used in the contact angle test. The spin coated casting solution was placed in the atmosphere for 20 minutes and then immersed into the water coagulation to obtain the mimetic porous matrix without dense skin layer, the nanoparticles could be uniformly dispersed in this matrix, which was a kind of ideal porous membrane matrix. Attenuated total reflectance fourier transform infrared spectroscopy (ATR-FTIR, Nicolet 8700, USA) was used to examine the surface composition of neat and hybrid PVDF membranes. Surface and cross-section morphology of neat and modified PVDF membranes were viewed with the field emission scanning electron microscope (FESEM, Hitachi SU8010, Japan), all the samples were coated with gold before observation. The porosity was calculated by measuring the weight of the wet and dry membrane. ${ }^{16}$ The pore size distribution of the prepared membranes was tested by the membrane pore size analyzer (GQ/PSMA-10, China). 


\subsection{Permeation experiments}

The separation performances of neat and modified PVDF membranes were investigated using a home-made filtration system with an effective membrane area of $12.5 \mathrm{~cm}^{2}$. The filtration experiments were carried out at $0.1 \mathrm{MPa}$ to obtain a stable flux by comparing the flux values every 10 minutes. The pure water flux was marked as $\mathrm{J}_{1}\left(\mathrm{~L} / \mathrm{m}^{2} \mathrm{~h}\right)$ and calculated by the following equation(1).The rejection $(\mathrm{R})$ of membrane was tested with BSA solution $(1 \mathrm{~g} / \mathrm{L}$, pH7.4) as feed solution and calculated according the equation (2), the concentrations of permeate and feed solution were examined via UV spectrophotometer (UV-1800, Shimadzu) with the characteristic wavelength at $280 \mathrm{~nm}$.

$$
\begin{gathered}
\mathrm{J}_{1}=\frac{\mathrm{V}}{\mathrm{A} \times \mathrm{t}} \\
\mathrm{R}=\left(1-\frac{C_{P}}{C}\right) \times 100 \%
\end{gathered}
$$

Where $\mathrm{V}(\mathrm{L})$ is the volume of permeated water, $\mathrm{t}(\mathrm{h})$ is the permeation time and $\mathrm{A}\left(\mathrm{m}^{2}\right)$ is the effective area of filtration. $\mathrm{Cp}$ and $\mathrm{C}$ are attributable to the BSA concentrations of permeate and feed solution.

\subsection{Fouling test}

The adsorption fouling test was executed using the neat and modified PVDF membranes. The tested membranes with regular shape were immersed into the pollutant solution (BSA, $0.5 \mathrm{~g} / \mathrm{L}, \mathrm{pH}$ 7.4). After oscillating incubation 24 hour to reach adsorption-desorption equilibrium at $37^{\circ} \mathrm{C}$, the concentrations of BSA solution before and after adsorption of PVDF membranes were measured with UV-1800 and the mass $\left(\mu \mathrm{g} / \mathrm{cm}^{2}\right)$ of adsorbed BSA on the membrane surface was calculated. In addition, the FITC-labeled BSA was prepared to investigate the anti-adsorption ability of modified membrane, the adsorbed BSA-FITC on the 
membrane surface was observed using a fluorescence microscope (QM/TM, PTI, USA).

For the filtration fouling, BSA (1g/L, $\mathrm{pH} 7.4)$ and $\mathrm{HA}(1 \mathrm{~g} / \mathrm{L})$ were used as two kinds of model pollutants. The fouled flux recovery ratio (FRR) and the fouled flux recovery ratio after washing (FRR-W) were employed to evaluate the antifouling properties of hybrid membranes. It should be notice that the membrane with the higher values of FRR and FRR-W indicated the better antifouling properties. The executed filtration fouling process consisted of three steps. First of all, stable flux $(\mathrm{J})$ was obtained by pure water filtration. Secondly, the feed solution was changed to the pollutant solution (BSA or HA) and another stable flux was obtained. Finally, pollutants solution was changed to pure water again and stable flux $\left(\mathrm{J}_{2}\right)$ was obtained, then a washing process was introduced and the membrane was taken out from the filter system to immerse in the phosphate butter for 20 minutes and rinsed with pure water for 10 minutes, and reinstalled back into filtration unit, the second water flux $\left(\mathrm{J}_{2 \mathrm{w}}\right)$ of pure water was recorded at the end. The FRR and FRR-W values were calculated by the equation (3) (4), respectively.

$$
\begin{aligned}
& \mathrm{FRR}=\frac{J_{2}}{J} \times 100 \% \\
& \mathrm{FRRW}=\frac{J_{2 w}}{J} \times 100 \%
\end{aligned}
$$

\section{Results and discussion}

The preparation procedure of $\mathrm{SiO}_{2}$-g-PDMS nanoparticles is shown in Fig.1. Based on the directional and quantitative reaction of hydroxyl and acyl chloride, acyl chloride groups are introduced to the HO-PDMS-OH segment by TMC, and employed to react with the hydroxyl of hydrophilic $\mathrm{SiO}_{2}$ nanoparticles based on the high reactivity of active groups. Theoretically, the prepared $\mathrm{SiO}_{2}$-g-PDMS nanoparticles should possess the special characteristics of 
hydrophobic shell and hydrophilic core as dual mode nanoparticles.

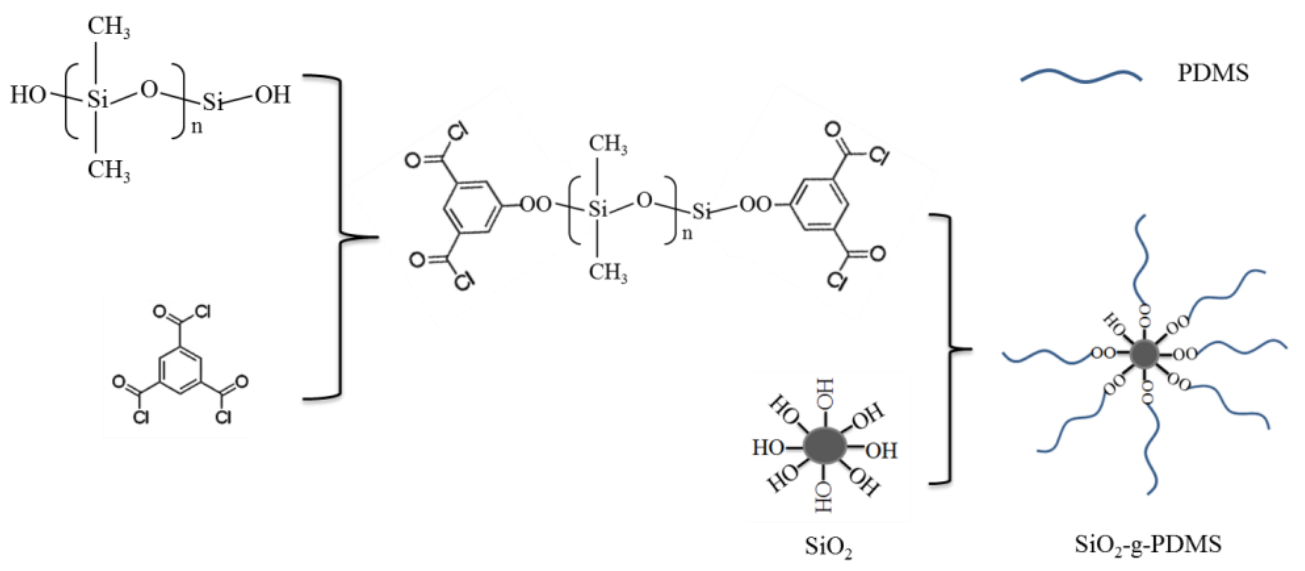

Fig. 1. Schematic illustration of the synthesis of $\mathrm{SiO}_{2}-\mathrm{g}-\mathrm{PDMS}$ nanoparticles.

a
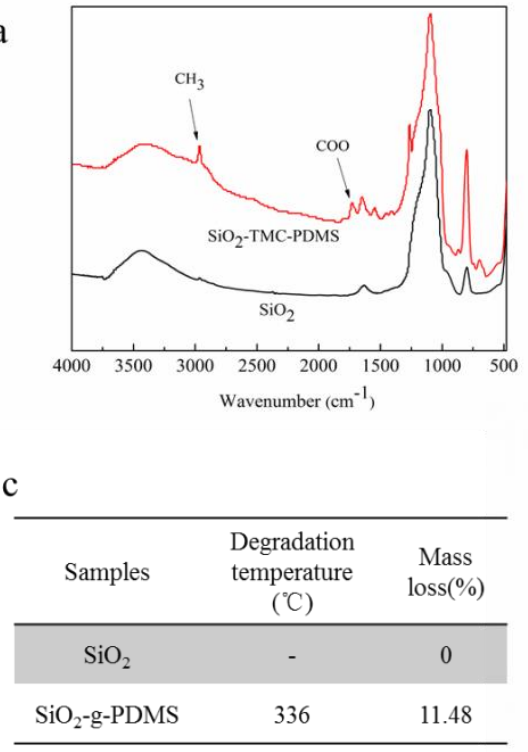

$\mathrm{b}$
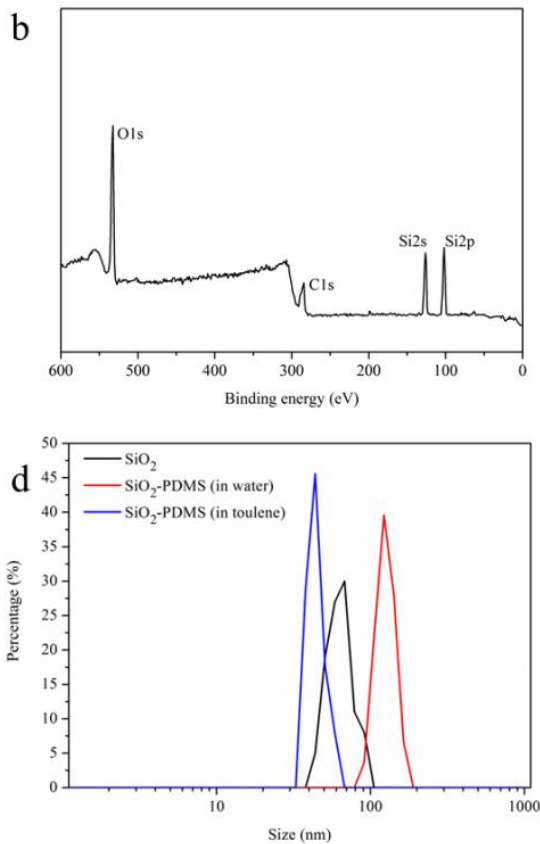

Fig. 2. Characterization of $\mathrm{SiO}_{2}$-g-PDMS nanoparticles (a: FTIR spectra. b: XPS spectra. c: TGA analysis. d: Particle size distribution).

The characterization results of modified $\mathrm{SiO}_{2}$-g-PDMS nanoparticles are shown in Fig.2. According to the IR spectra of $\mathrm{SiO}_{2}$ nanoparticles before and after modification (Fig.2a), the new characteristic absorption peaks at $1730 \mathrm{~cm}^{-1}$ and $2960 \mathrm{~cm}^{-1}$ appear in the spectrum of $\mathrm{SiO}_{2}$-g-PDMS nanoparticles. The $1730 \mathrm{~cm}^{-1}$ peak is the characteristic absorption peak of ester 
bond, which is assigned to the reaction product of the terminal hydroxyl of HO-PDMS-OH or $\mathrm{SiO}_{2}$ and acyl chloride group of TMC, suggesting the existence of TMC in the $\mathrm{SiO}_{2}$-g-PDMS nanoparticles. The $2960 \mathrm{~cm}^{-1}$ peak belongs to the stretching vibration of methyl in HO-PDMS-OH. So it is clear that the PDMS and TMC are successfully grafted onto the surface of $\mathrm{SiO}_{2}$ nanoparticles by the reaction of esterification. Fig.2b shows the XPS spectra of $\mathrm{SiO}_{2}$-g-PDMS nanoparticles, the distinctly $\mathrm{C}$ element belongs to the TMC molecules and PDMS, which differs from the sole $\mathrm{Si}$ and $\mathrm{O}$ elements of $\mathrm{SiO}_{2}$ spectrum. Fig. 2c exhibits the TGA analysis result of $\mathrm{SiO}_{2}$-g-PDMS and $\mathrm{SiO}_{2}$ nanoparticles, the thermal degradation occurs at $336^{\circ} \mathrm{C}$ with the mass loss of $11.48 \%$, which is due to the decomposition of grafted PDMS segments on $\mathrm{SiO}_{2}$ nanoparticles, and the mass loss are not observed from pristine $\mathrm{SiO}_{2}$ nanoparticles. Fig. 2d shows the particle size distribution of $\mathrm{SiO}_{2}$ nanoparticles before and after modification in different solvents. It can be seen that the average diameter of $\mathrm{SiO}_{2}$-g-PDMS nanoparticles in toluene is about $42 \mathrm{~nm}$, suggesting a more narrow distribution range than pristine $\mathrm{SiO}_{2}$ nanoparticles. However, the average diameter of $\mathrm{SiO}_{2}$-g-PDMS nanoparticles in water is about $135 \mathrm{~nm}$. These changed sizes of $\mathrm{SiO}_{2}$-g-PDMS nanoparticles in different solvent are similar to the self-assembly feature of amphiphilic polymer in good solvent and poor solvent. ${ }^{17,18}$ It can be concluded that the different size of $\mathrm{SiO}_{2}$ nanoparticles may be due to the shell-core structure of $\mathrm{SiO}_{2}$-g-PDMS nanoparticles. PDMS segment with the strong hydrophobicity on $\mathrm{SiO}_{2}$-g-PDMS nanoparticles surface will exhibit crimped state in the poor solvent of water, the self-assembly effect of PDMS makes several $\mathrm{SiO}_{2}$-g-PDMS particles get together resulting in the increase of the dynamic diameter of $\mathrm{SiO}_{2}$-g-PDMS nanoparticles. The PDMS segments with extended state are able to improve the dispersion of 
$\mathrm{SiO}_{2}$-g-PDMS nanoparticles to decrease the dynamic diameter of nanoparticles in the good solvent of toluene. Moreover, these results also prove the successful preparation of $\mathrm{SiO}_{2}$-g-PDMS nanoparticles. It should be noted that the diameter of the $\mathrm{SiO}_{2}$ nanoparticles in water is greater than the labeled value of raw material due to the agglomeration problem.

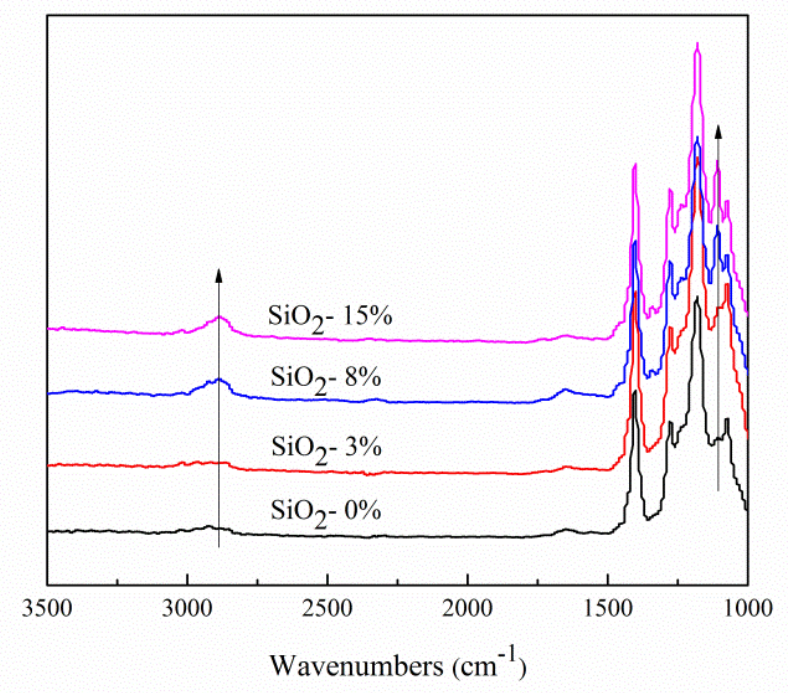

Fig.3. ATR-FTIR spectra of neat and modified PVDF membranes.

In order to investigate the impact of adding $\mathrm{SiO}_{2}$-g-PDMS nanoparticles on the surface chemical composition of PVDF membrane, ATR-FTIR is employed to evaluate the change of surface composition, and the spectra of neat and hybrid PVDF membranes are shown in Fig.3. Obviously, the characteristic absorption peaks of PVDF are all below $1500 \mathrm{~cm}^{-1}$, and there is no significant difference in the spectra of PVDF and 3\% membranes. With the increasing amount of added $\mathrm{SiO}_{2}$-g-PDMS nanoparticles, the distinctly absorption peaks at $1103 \mathrm{~cm}^{-1}$ appear in the spectra of $8 \%$ and $15 \%$ membranes, belonging to the $\mathrm{Si}-\mathrm{O}$ bond. In addition, the absorption peak of $2916 \mathrm{~cm}^{-1}$ is assigned to the stretching vibration of methyl in the PDMS segments, indicating the existence of $\mathrm{SiO}_{2}$-g-PDMS nanoparticles in the skin layer of the PVDF membrane based on the detection depth of ATR-FTIR. 
Fig. 4 shows the SEM images of membrane surface and cross section of hybrid PVDF membranes. According to the images of A, C, E and G, a large number of pores with the diameters of several tens of nanometers can be observed on the membrane surface, and the number of membrane pore increases with the increasing amount of added $\mathrm{SiO}_{2}$-g-PDMS nanoparticles, but it should be noted that there are no visible nanoparticles on the membrane surface. The hybrid membranes exhibit an asymmetric structure of dense skin layer and the finger like support layer viewed from the images of $\mathrm{B}, \mathrm{D}, \mathrm{F}, \mathrm{H}$, and there is no large-scale reunion problem of nanoparticles. As for the image of reference sample (I, J), a large number of agglomerated nanoparticles can be observed in the cross-section image. These SEM results show that adding $\mathrm{SiO}_{2}$-g-PDMS nanoparticles do not affect the membrane structure, and help to increase the number of membrane pores without changing the skin layer. To further investigate the effect of addition of $\mathrm{SiO}_{2}$-g-PDMS nanoparticles to the membrane structure, the porosity and average pore sizes of neat and hybrid PVDF membranes are shown in Table 1. Obviously, adding $\mathrm{SiO}_{2}$-g-PDMS nanoparticles is conducive to increase the porosity and pore sizes of hybrid PVDF membranes

Table 1 Porosity and pore sizes of all the prepared PVDF membranes.

\begin{tabular}{ccc}
\hline Samples & Porosity $(\%)$ & $\begin{array}{r}\text { Average pore } \\
\text { size }(\mathrm{nm})\end{array}$ \\
\hline PVDF & $78( \pm 3)$ & 12.2 \\
$3 \%$ & $81( \pm 4)$ & 13.1 \\
$8 \%$ & $82( \pm 1)$ & 13.6 \\
$15 \%$ & $88( \pm 4)$ & 16.4 \\
\hline
\end{tabular}




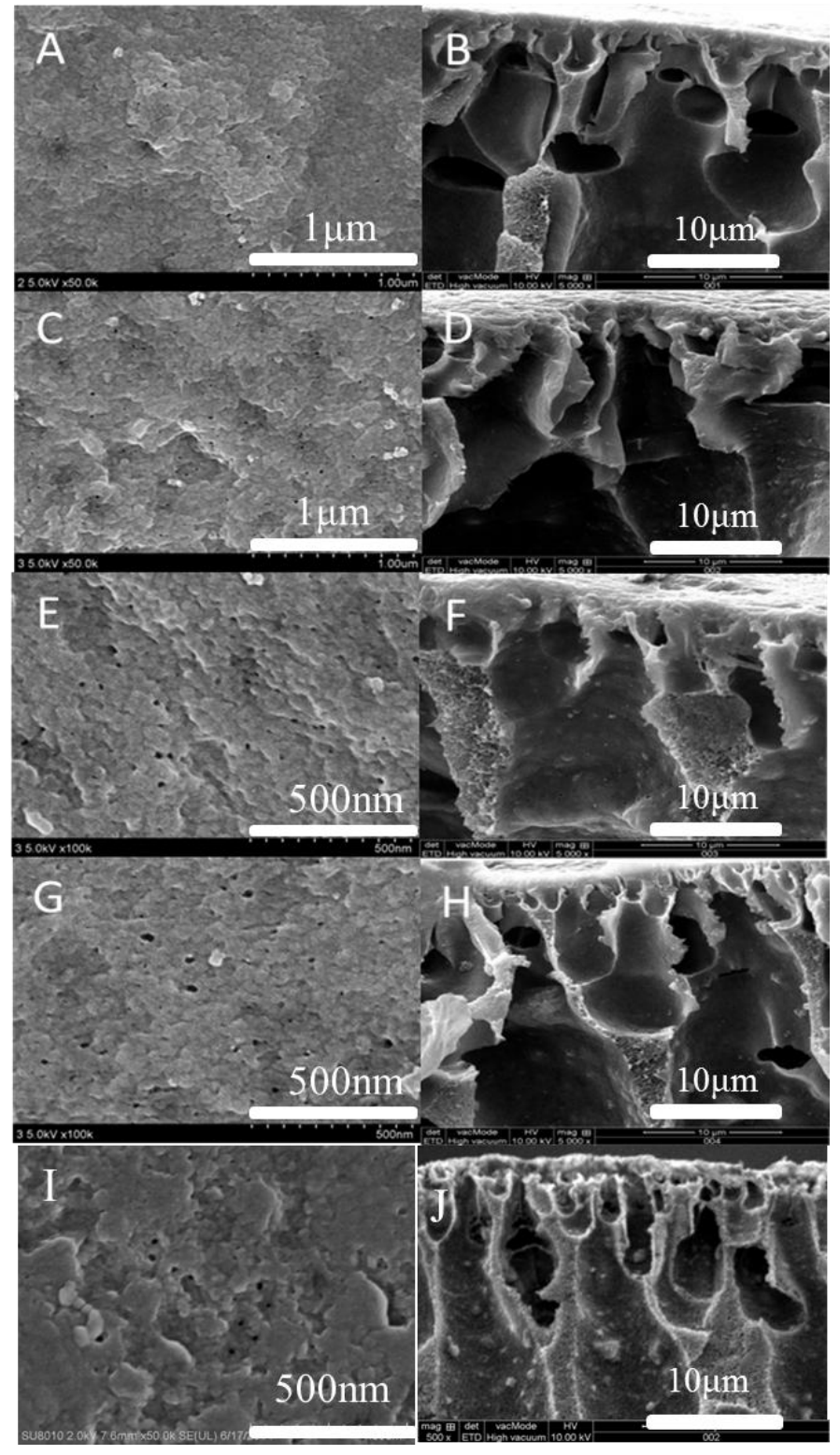

Fig.4. SEM images of neat and modified PVDF membranes (A, C, E, G, I: surface images. B, D, F, H, J: cross section. A, B: PVDF membrane. C, D: 3\% membrane. E, F: $8 \%$ membrane. G, $\mathrm{H}: 15 \%$ membrane. I, J: the reference sample with $8 \% \mathrm{SiO}_{2}$ ).

The wetting property of membrane surface is directly related to the separation performance and antifouling property, the CA is used to estimate the wetting ability of membrane surface and mimetic matrix of all the PVDF membranes. As shown in Fig.5a, the CA of PVDF, $3 \%, 8 \%$ and $15 \%$ membrane are $84^{\circ}, 93^{\circ}, 114^{\circ}$ and $121^{\circ}$, respectively. The data of Fig. $5 \mathrm{~b}$ indicates the added $\mathrm{SiO}_{2}$-g-PDMS nanoparticles can significantly improve the 
hydrophilicity of internal porous structure with the CA decreasing to $15^{\circ}$.

Obviously, the CA values of the PVDF membrane matrix and surface exhibit different variation trend after blending $\mathrm{SiO}_{2}$-g-PDMS nanoparticles. According to the ATR-FTIR and SEM results, the $\mathrm{SiO}_{2}$-g-PDMS nanoparticles are embedded in the skin layer of membrane surface and exposed in the porous matrix, and PDMS segments are enriched on the hybrid membrane surface. Therefore, the increased CA values of hybrid PVDF membrane surface should be attributed to the enriched PDMS segments due to the difference in interfacial free energy of PDMS and PVDF segments. The decreased CA values of mimetic matrix should be attributed to the exposed $\mathrm{SiO}_{2}$-g-PDMS nanoparticles, which is still able to exhibit excellent hydrophilicity of $\mathrm{SiO}_{2}$ to improve the wettability of PVDF membrane matrix.
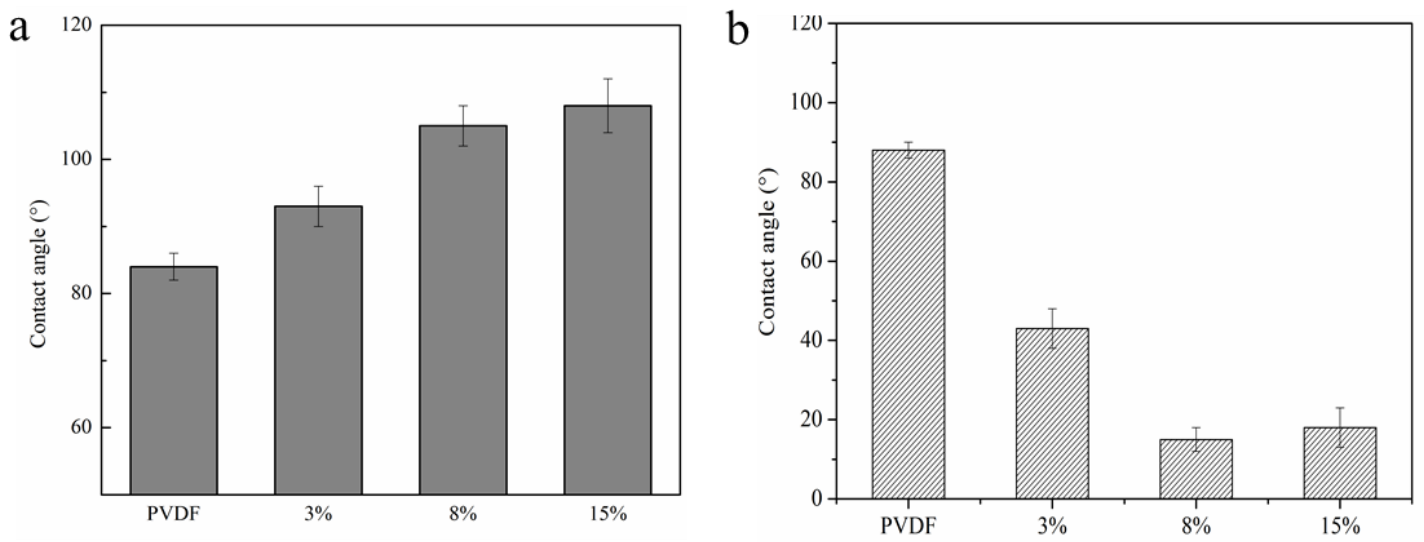

Fig.5. Contact angle of neat and modified PVDF membranes (a: surface, b: mimetic matrix).



Fig. 6. Schematic diagram of PVDF hybrid membrane. 
According to the above evaluation of ATR, CA and SEM, it can be inferred the dual mode nanoparticles provide assistance for the hybrid modification of PVDF membrane. The presence of hydrophobic PDMS segments will help to improve the dispersion effect of hydrophilic particles in the hydrophobic matrix, the presence of hydrophilic nanoparticles are conducive to increase the chances of forming membrane pores by delaying solvent exchange and improve the wetting properties of the PVDF membrane, which provides the foundation for the optimization of the separation and antifouling properties. According to the above characterization results, it can be inferred that the cross-section structure of hybrid membrane should look like the diagram of Fig. 6. The $\mathrm{SiO}_{2}$ nanoparticles are dispersed in the membrane matrix and skin layer, and PDMS segments enrich towards the membrane surface.

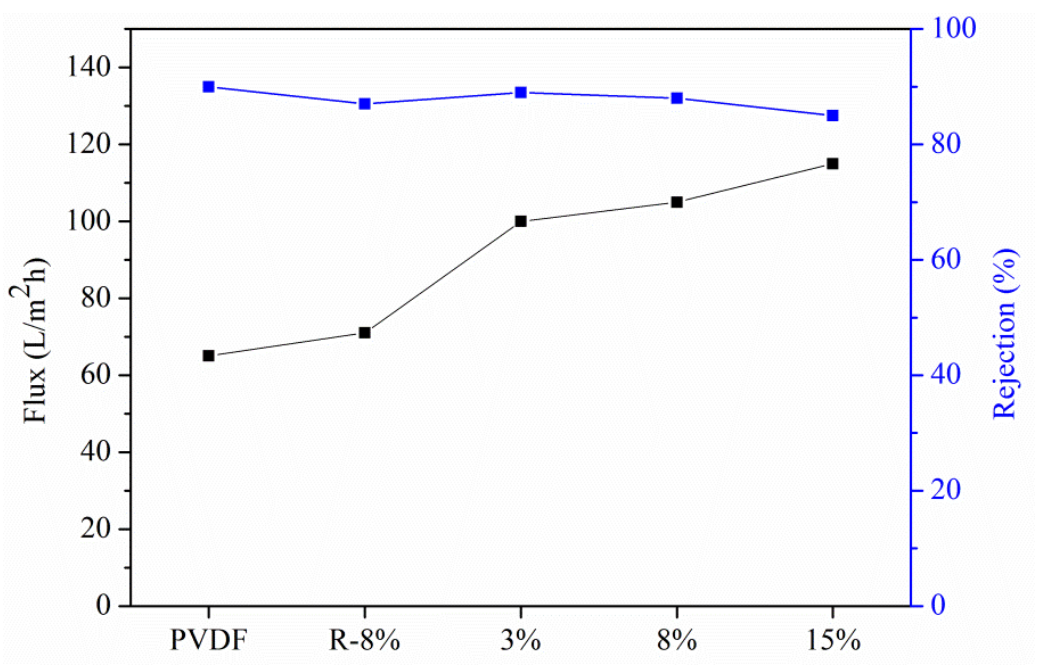

Fig.7. Separation performance of neat and hybrid PVDF membranes.

Fig. 7 shows the separation performance of neat and hybrid PVDF membranes. Due to the added $\mathrm{SiO}_{2}$-g-PDMS nanoparticles, the water flux of hybrid membrane increases from 65 $\mathrm{L} / \mathrm{m}^{2} \mathrm{~h}$ to $115 \mathrm{~L} / \mathrm{m}^{2} \mathrm{~h}$, and there is no striking difference in the rejection of BSA. Under the same operating pressure, the improved hydrophilicity and increased membrane pores are 
conducive to accelerate the permeation of water molecules to increase the water flux, and the stable rejection suggests adding $\mathrm{SiO}_{2}$-g-PDMS nanoparticle will not undermine the effective separation skin layer of PVDF membrane as shown in Fig.4. It is worth noticed that there is no significant improvement in the separation performance between the reference sample (R-8\%) and neat PVDF membrane, due to the significant reunion problem of $\mathrm{SiO}_{2}$ nanoparticles.

a

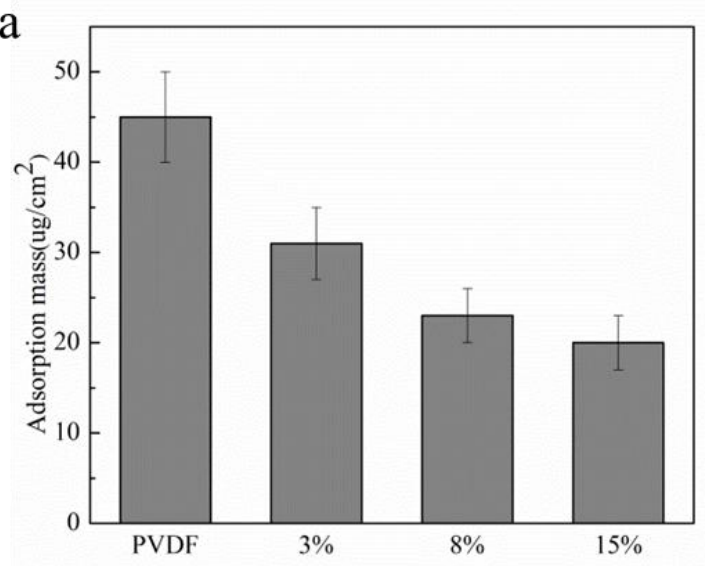

$\mathrm{b}$
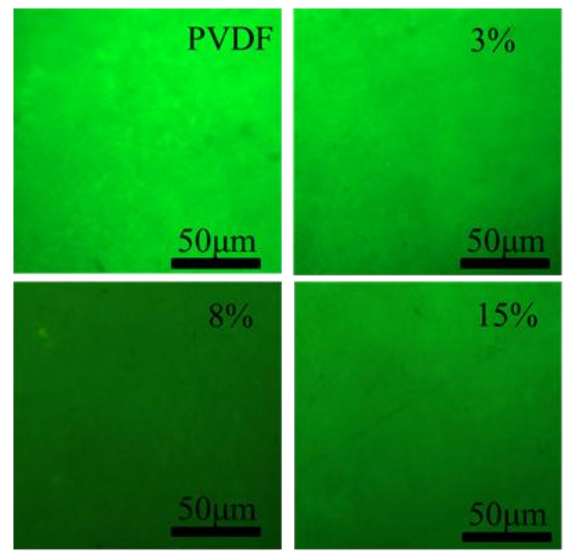

Fig.8. Anti-adsorption ability of membranes (a: adsorption mass of BSA on membranes surface. b: fluorescence microscopy images of fouled membranes).

In order to evaluate the antifouling properties of hybrid PVDF membrane, the pollutant adsorption test and dynamic fouling test were employed in this work. Fig. 8a shows the anti-adsorption capacity of hybrid PVDF membranes, the adsorption mass of BSA on the membrane surface decrease from $45 \mu \mathrm{g} / \mathrm{cm}^{2}$ to $19 \mu \mathrm{g} / \mathrm{cm}^{2}$ with increasing the added content of $\mathrm{SiO}_{2}$-g-PDMS nanoparticle from $0 \%$ to $15 \%$, it is clear that the added $\mathrm{SiO}_{2}$-g-PDMS nanoparticles in skin layer are able to ease the adsorption of BSA due to the surface aggregation of active PDMS segments with fouling release ability, because even the adsorbed BSA can be released in oscillating incubation by PDMS segments. Fig. $8 \mathrm{~b}$ presents the 
fluorescence microscopy images of neat and modified PVDF membranes after the adsorption test of fluorescence-labeled BSA solution. Obviously, the image of neat PVDF membrane exhibits the bright green, while the images of modified membranes show the dark green with decreased fluorescence intensity, indicating the less BSA-FITC adsorbed on the modified membrane surface were suppressed due to the decreased surface energy by PDMS segments.
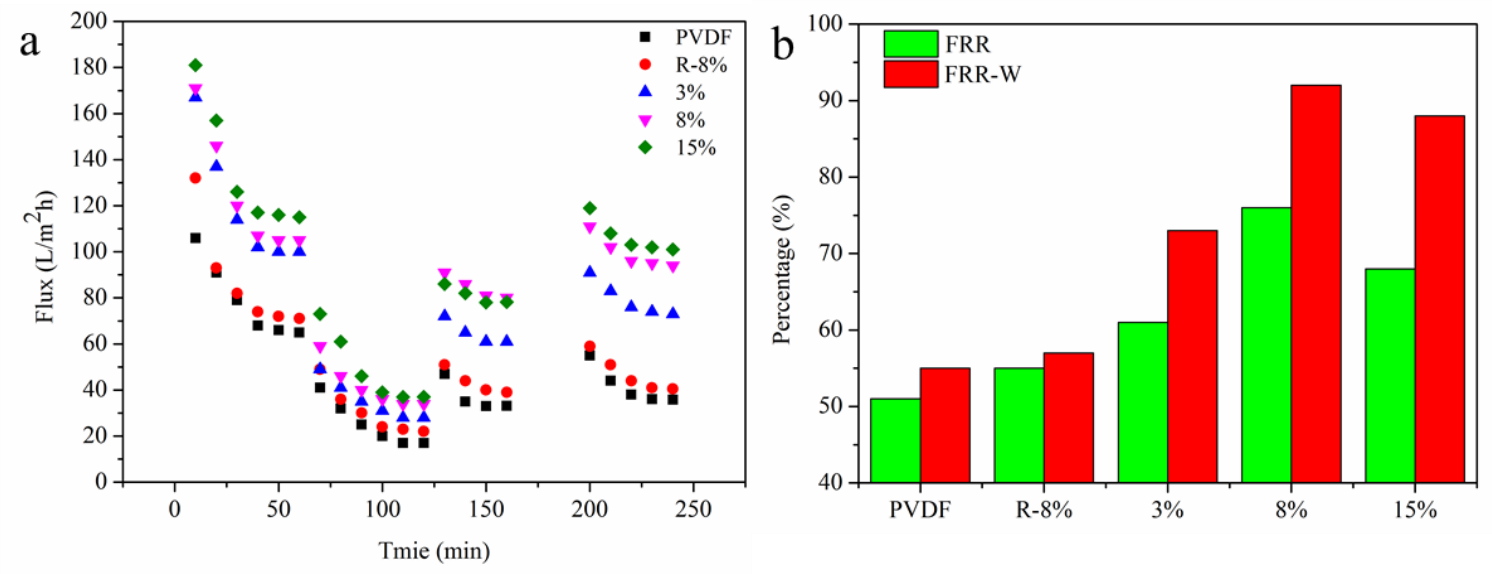

Fig.9. Filtration fouling test of neat and modified PVDF membranes using BSA as pollutant (0-60min, water, 61-120min, BSA solution, 121-160min, water, 161-190min, cleaning process, 191-240min, water).
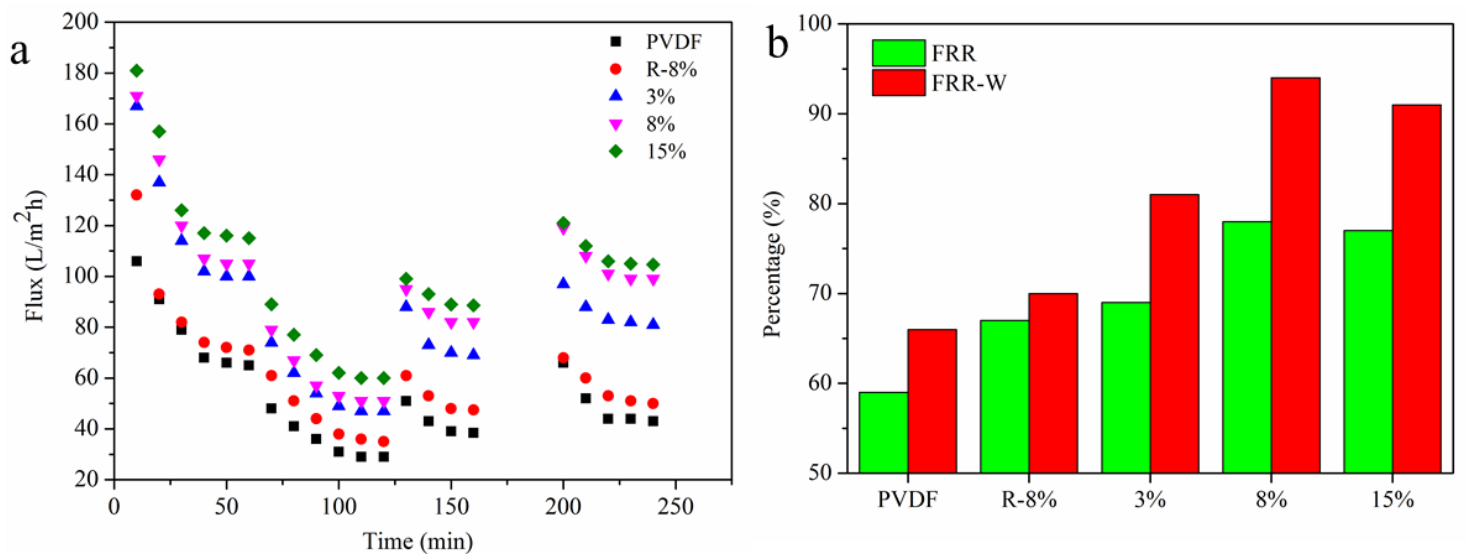

Fig.10. Filtration fouling test of neat and modified PVDF membranes using HA as pollutant (a: 0-60min, water, 61-120min, BSA solution, 121-160min, water, 161-190min, cleaning process, 191-240min, water. b: FRR values). 
As far as we know, the fouled membrane with the higher values of FRR and FRR-W indicates the better antifouling properties, and the difference of FRR and FRR-W value represents the reversible ratio of fouling. Fig. 9 exhibits the dynamic fouling test using BSA as pollutant. According to the FRR values in Fig. 9b, even without washing process, the FRR values of $3 \%, 8 \%$ and $15 \%$ membranes are much higher than the $51 \%$ of PVDF membrane, showing the improved ability of fouling repulsion due to the reduced amount of adsorbed pollutants clogging the membrane pores to decrease the water flux. After the washing process, the FRR-W values of hybrid $3 \%, 8 \%$ and $15 \%$ membranes increase to $73 \%, 92 \%$ and $88 \%$. The promoted proportions of FRR-W values are all significantly higher than that of PVDF membrane, showing an improved ability of fouling release. The reason may be attributed to the dual-mode antifouling effect of $\mathrm{SiO}_{2}$-g-PDMS nanoparticles. The hydrophilic core of $\mathrm{SiO}_{2}$-g-PDMS nanoparticles can enhance the hydrophilicity of matrix structure of PVDF membrane, based on the principle of the interfacial hydration layer as protective barrier, ${ }^{3,19}$ the effectively suppressed hydrophobic interaction between PVDF and pollutants BSA is conducive to improve the fouling repulsion ability of PVDF membrane materials. The existed PDMS segments with the very low surface energy and lubrication performance, is also able to weaken the anchoring effect of BSA in the interface of PVDF membrane, so higher FRR-W value can be obtained because pollutants are more easily cleaned due to the shear force during the water washing process. Fig. 10 shows the dynamic fouling test result of HA, which is a natural pollutant in sewage. ${ }^{20}$ The FRR and FRR-W values of hybrid PVDF membrane exhibit similar trends with the test results of BSA and the FRR-W value of $8 \%$ increases to $94 \%$, suggesting the added $\mathrm{SiO}_{2}$-g-PDMS nanoparticle also can remit the 
irreversible fouling problem caused by HA. These results demonstrate that the probability of various pollutants adsorbed and deposited in the hybrid membrane pores and membrane surface is effectively decreased, and a higher removal probability of pollutant is obtained based on the dual mode antifouling property of $\mathrm{SiO}_{2}$-g-PDMS nanoparticles with fouling repulsion and fouling release ability, the irreversible fouling caused by the anchored pollutants is significantly decreased. ${ }^{21,22}$ Therefore, the hybrid PVDF membranes are able to show the better antifouling property.

\section{Conclusions}

The hybrid PVDF membranes are prepared though NIPs method with the dual-mode $\mathrm{SiO}_{2}$-g-PDMS nanoparticles as modifier. The efficient hybrid of $\mathrm{SiO}_{2}$-g-PDMS nanoparticles with the hydrophilic core and hydrophobic shell is able to enhance the separation performance and the antifouling ability of PVDF membranes. Based on the release and repulsion ability of $\mathrm{SiO}_{2}$-g-PDMS nanoparticles, the modified PVDF membranes exhibit improved anti-adsorption ability during adsorption fouling test, and the FRR-W values of modified $8 \%$ membrane are both more than $90 \%$ for the two typical pollutants of BSA and HA during filtration fouling. It can be inferred the better performance will be obtained when the multifunctional nanoparticles are used for hybrid modification.

\section{Acknowledgements}

This work is supported by grants from the Program for New Century Excellent Talents in University (No.NCET-12-0827), Program of Introducing Talents of Discipline to Universities (No.111-2-04) and Innovation Funds for $\mathrm{PhD}$ Students of Donghua University (CUSF-DH-D-2016037). 


\section{References}

1 M. A. Shannon, P. W. Bohn, M. Elimelech, J. G. Georgiadis, B. J. Marinas and A.M. Mayes, Science and technology for water purification in the coming decades, Nature 2008, 452, 301.

2 D. Rana and T. Matsuura, Surface modifications for antifouling membranes, Chem. Rev. $2010,110,2448$

3 W. Chen, Y. Su, J. Peng, X. Zhao, Z. Jiang, Y. Dong, Y. Zhang, Y. Liang and J. Liu, Efficient wastewater treatment by membranes through constructing tunable antifouling membrane surfaces, Environ. Sci. Technol. 2011, 45, 6545.

4 Y. F. Yang, Y. Li, Q. L. Li, L. S. Wan and Z. K. Xu, Surface hydrophilization of microporous polypropylene membrane by grafting zwitterionic polymer for anti-biofouling, J. Membr. Sci. 2010, 362, 255.

5 X. T. Zhao, W. J. Chen, Y. L. Su, W. Zhu, J. M. Peng and Z. Y. Jiang, Hierarchically engineered membrane surfaces with superior antifouling and self-cleaning properties, J. Membr. Sci. 2013, 441, 93.

6 I. Banerjee, R. C. Pangule and R. S. Kane, Antifouling coatings: recent developments in the design of surfaces that prevent fouling by proteins, bacteria, and marine organisms, Adv. Mater. 2011, 23, 690.

7 M. Kobayashi, Y. Terayama, H. Yamaguchi, M. Terada, D. Murakami, K. Ishihara and A. Takahara, Wettability and antifouling behavior on the surfaces of superhydrophilic polymer brushes, Langmuir 2012, 28, 7212.

8 Z. G. Estephan, P. S. Schlenoff and J. B. Schlenoff, Zwitteration as an alternative to pegylation, Langmuir 2011, 27, 6794.

9 J. B. Schlenoff, Zwitteration: Coating surfaces with zwitterionic functionality to reduce nonspecific adsorption, Langmuir 2014, 30, 9625-9636.

10 I. Banerjee, R.C. Pangule, R.S. Kane, Antifouling coatings: recent developments in the design of surfaces that prevent fouling by proteins, bacteria, and marine organisms. Adv. Mater. 2011, 23,690-718.

11 Y. Chang, C.Y. Ko, Y. J. Shih, D. Quemener, A. Deratani, T. C. Wei, D. M. Wang and J. Y. 
Lai, Surface grafting control of PEGylated poly(vinylidene fluoride) antifouling membrane via surface-initiated radical graft copolymerization, J. Membr. Sci., 2009, 345, 160.

12 S. F. Chen, J. Zheng, L. Y. Li and S. Y. Jiang, Strong Resistance of Phosphorylcholine Self-assembled Monolayers to Protein Adsorption: Insights into Nonfouling Properties of Zwitterionic Materials, J. Am. Chem. Soc. 2005, 127, 14473.

13 L. L. Zhou, W. Z. Yuan, J. Y. Yuan and X. Y. Hong, Preparation of double-responsive $\mathrm{SiO}_{2}$-g-PDMAEMA nanoparticles via ATRP, Mater. Lett. 2008, 61, 1372.

14 X. T. Zhao, Y. L. Su, Y. F. Li, R. N. Zhang, J. J. Zhao and Z. Y. Jiang, Engineering amphiphilic membrane surfaces based on PEO and PDMS segments for improved antifouling performances, J. Membr. Sci. 2014, 450, 111.

15 J. E. Mark, Some interesting things about polysiloxanes, Acc. Chem. Res. 2004, 37, 946.

16 A. Qin, X. Li, X. Zhao, D. Liu, C. He, Preparation and characterization of nano-chitin whisker reinforced PVDF membrane with excellent antifouling property. J. Membr. Sci. $2015,480,1-10$

17 B. L. Guo, A. F. Wistrand and A. C. Albertsson, Simple route to size-tunable degradable and electroactive nanoparticles from the self-assembly of conducting coil-rod-coil triblock copolymers, Chem. Mater. 2011, 23, 4045.

18 S. Basu, D. Rao Vutukuri, S. Shyamroy, B. S. Sandanaraj and S. Thayumanavan, Invertible amphiphilic homopolymers, J. Am. Chem. Soc. 2004, 126, 9890.

19 J. B. Schlenoff, Zwitteration: Coating surfaces with zwitterionic functionality to reduce nonspecific adsorption, Langmuir 2014, 30, 9625.

20 X. R. Meng, W. T.Tang, L. Wang, X. D. Wang, D. X. Huang, H. N. Chen and N. Zhang, Mechanism analysis of membrane fouling behavior by humic acid using atomic force microscopy: Effect of solution $\mathrm{pH}$ and hydrophilicity of PVDF ultrafiltration membrane interface, J. Membr. Sci. 2015, 487, 180.

21 S. Liang, K. Xiao, Y. H. Mo and X. Huang, A novel ZnO nanoparticle blended polyvinylidene fluoride membrane for anti-irreversible fouling, J. Membr. Sci. 2012, 394, 184.

22 Y. Q. Wang, T. Wang, Y. L. Su, F. B. Peng, H. Wu and Z.Y. Jiang, Remarkable reduction 
of irreversible fouling and improvement of the permeation properties of poly(ether sulfone) ultrafiltration membranes by blending with pluronic F127, Langmuir 2005, 21, 11856. 